\title{
Tyrosinase:146-156 Peptide
}

National Cancer Institute

\section{Source}

National Cancer Institute. Tyrosinase:146-156 Peptide. NCI Thesaurus. Code C48630.

A synthetic peptide consisting of amino acid residues 146 through 156 of the enzyme tyrosinase, a protein frequently expressed by melanoma cells, with antitumor activity. Vaccination with tyrosinase:146-156 peptide may stimulate cytotoxic T lymphocyte response against tyrosinase-expressing tumor cells, resulting in decreased tumor growth and cell lysis. 